\title{
PRODUÇÃO DE CONHECIMENTO EM UM CURSO DE MESTRADO EM ENFERMAGEM NO BRASIL ${ }^{1}$
}

\author{
KNOWLEDGE PRODUCTION IN A BRAZILIAN MASTER'S \\ DEGREE COURSE IN NURSING
}

\section{PRODUCCIÓN DEL CONOCIMIENTO EN UN CURSO DE MAESTRÍA EN ENFERMERÍA EN BRASIL}

\author{
Marta Lenise do Prado* \\ Patricia Kuerten Rocha ${ }^{* *}$ \\ VANia Marli Schubert Backes ${ }^{* * *}$ \\ KENYA S. REIBNITZ ${ }^{* * * *}$ \\ ROBERTA WATERKEMPER ${ }^{* * * * *}$ \\ Diana Coelho Gomes ${ }^{* * * * *}$
}

\begin{abstract}
RESUMO
Trata da análise documental, quantitativa-descritiva, das 60 dissertações defendidas no Curso de Mestrado do Programa de Pós-graduação em Enfermagem (PEn) da Universidade Federal de Santa Catarina - UFSC, Brasil, de 2005 a 2008. Teve por objetivo conhecer algumas características do conhecimento produzido no Curso de Mestrado, no tocante a área temática, tipo de estudo, linhas de pesquisa e participantes dos estudos. Os dados foram coletados mediante a leitura dos resumos do texto completo das dissertações, arquivadas no PEN/ UFSC. Para o registro dos dados foi elaborado um instrumento para classificar as dissertações quanto às variáveis em estudo. Os dados foram analisados por meio de medidas estatísticas (números absolutos e relativos) e apresentados em forma de tabelas e gráficos. Os resultados evidenciam a consolidação de um conhecimento voltado para o cuidado à saúde e para a educação e a predominância de estudos qualitativos, distribuídos predominantemente em três linhas de pesquisa: O cuidado e processo de viver, ser saudável e adoecer, Educação, saúde e enfermagem e Processo de trabalho em saúde e enfermagem. Com relação aos participantes evidenciou-se a predominância de enfermeiros e da equipe de enfermagem, seguidos por familiares/ acompanhantes/ clientes e mulheres e gestantes como sujeitos envolvidos nos estudos.
\end{abstract}

Palavras chave: Enfermagem, produção de conhecimento, educação superior, avaliação.

\footnotetext{
${ }^{1}$ Artigo originado da pesquisa "Tecnologias convergentes-assistenciais em enfermagem: a contribuição de um Curso de Mestrado" com apoio do Conselho Nacional de Desenvolvimento Científico e Tecnológico.

*Enfermeira, professora do Departamento de Enfermagem e do Programa de Pós-graduação em Enfermagem da Universidade Federal de Santa Catarina (UFSC). Membro do Grupo de Pesquisa Educação, Saúde e Enfermagem - EDEN. Pesquisadora do CNPq. Florianópolis. Santa Catarina, Brasil. Email: mpradop@ccs.ufsc.br

${ }^{* *}$ Enfermeira, professora do Departamento de Enfermagem da Universidade Federal de Santa Catarina (UFSC), Membro do Grupo de Pesquisa GIATE. Florianópolis, Santa Catarina, Brasil. Email: patricia@ccs.ufsc.br

${ }^{* * *}$ Enfermeira, professora do Departamento de Enfermagem e do Programa de Pós-graduação em Enfermagem da Universidade Federal de Santa Catarina (UFSC). Membro do Grupo de Pesquisa Educação, Saúde e Enfermagem - EDEN. Pesquisadora do CNPq. Florianópolis, Santa Catarina, Brasil. Email: oivania@ccs.ufsc.br

${ }_{* * * *}$ Enfermeira, professora do Departamento de Enfermagem e do Programa de Pós-graduação em Enfermagem da Universidade Federal de Santa Catarina (UFSC). Membro do Grupo de Pesquisa Educação, Saúde e Enfermagem - EDEN. Florianópolis, Santa Catarina, Brasil. Email: kenya@ccs.ufsc.br

***** Enfermeira, doutoranda do Programa de Pós-graduação em Enfermagem da Universidade Federal de Santa Catarina - UFSC. Membro do Grupo de Pesquisa Educação, Saúde e Enfermagem - EDEN. Bolsista CNPq. Florianópolis, Santa Catarina, Brasil. Email: robswater@yahoo.com.br

${ }_{* * * * * *}$ Enfermeira pela Universidade Federal de Santa Catarina, Bolsista IC/CNPq. Membro do Grupo de Pesquisa Educação Saúde e Enfermagem - EDEN. Brasil.Email: alodiana@yahoo.com.br
} 


\begin{abstract}
This is a descriptive, quantitative documentary analysis, considering 60 theses defended at the Master Degree Course from the Graduate Program in Nursing (PEN) of the Federal University of Santa Catarina - UFSC, Brazil, from 2005 to 2008. Its purpose was to know some knowledge's characteristics produced at the Master's course in relation to the subject area, type of study, research agenda and study participants. Data were collected by reading the abstracts, full text of theses, available on file at the PEN / UFSC. For data recording a tool was developed for classifying the study variables. Data were analyzed using statistical measures (absolute and relative numbers) and presented in tables and graphs. Results show the consolidation of knowledge addressed to health care and education as well as the predominance of qualitative studies, distributed predominantly in three lines of research: The care and the process of living, being healthy and sick, education, health and nursing work process in health and nursing. As for subjects of study the predominance of nurses and nursing staff, were identified followed by relatives / escorts / clients, and pregnant women, as well.
\end{abstract}

Key words: Nursing, knowledge production, higher education, evaluation.

\title{
RESUMEN
}

Se trata de un análisis documental, cuantitativo-descriptivo, de las 60 tesis defendidas en el Curso de Maestría del Programa de Posgrado en Enfermería (PEn) de la Universidad Federal de Santa Catarina - UFSC, Brasil, de 2005 a 2008. Tuvo por objetivo conocer algunas características del conocimiento producido en el curso de Maestría, en relación al área temática, tipo de estudio, líneas de investigación y participantes de los estudios. Los datos fueron recolectados mediante la lectura de los resúmenes y texto completo de las tesis disponibles en archivo en el PEN/ UFSC. Para el registro de los datos fue elaborado un instrumento para clasificar las tesis en base a las variables de estudio. Los datos fueron analizados por medio de medidas estadísticas (números absolutos y relativos) y presentados en forma de tablas y gráficos. Los resultados evidencian la consolidación del conocimiento dirigido al cuidado en salud y la educación, así como el predominio de estudios cualitativos, distribuidos predominantemente en tres líneas de investigación: El cuidado y el proceso de vivir, ser saludable y enfermar; educación, salud y enfermería; proceso de trabajo en salud y enfermería. En cuanto a los sujetos de estudio, se identificó el predominio de enfermeros y del equipo de enfermería, seguido por familiares/ acompañantes/ clientes, además de mujeres y embarazadas.

Palabras clave: Enfermería, producción de conocimiento, educación superior, evaluación.

Fecha recepción: 01/01/11 Fecha aceptación: 28/11/11

\section{INTRODUÇÃO}

O corpo de conhecimento da Enfermagem vem sendo construído sistematicamente a partir de esforços de muitas enfermeiras ao longo da história da profissão. Muitas descobertas e conquistas de enfermeiras como Catherine McAuley que conjuntamente com outras enfermeiras irlandesas, no começo do Século 19, fundaram em Dublin o "Institute of Our Lady Mercy", estabelecendo serviços de enfermagem domiciliar e hospitalar, contribuíram para essa construção (1). Também constituem contribuições importantes os escritos e descobertas de Florence Nightingale (1859), que expressam o significado do processo de cuidado, a conceptualização de ambiente, a advertência para as enfermeiras não focarem somente a doença e a diferenciação do enfoque teórico e do objeto de estudo da Enfermagem do da Medicina, e estabelecem diretrizes para a prática da Enfermagem (27). 
No Brasil, o impulso na produção do corpo de conhecimento da Enfermagem se dá a partir da década de 20 quando cerca de dezessete enfermeiras diplomadas pela Escola de Enfermagem Anna Nery realizaram cursos de pós-graduação nos Estados Unidos, com bolsa de estudos da Fundação Rockfeller, sendo que nas décadas de 40 e de 50, o espectro de brasileiras em cursos de pósgraduação no exterior foi significativo (8). Ao longo do Século XX, a expansão dos cursos de graduação e a implantação de cursos de pós-graduação strictu-sensu - mestrado e doutorado, contribuem sobremaneira para o incremento da produção científica da Enfermagem brasileira (9).

Ainda hoje, a produção do conhecimento em enfermagem no Brasil está fortemente associado à formação em nível de mestrado e doutorado, e esta consiste numa importante estratégia de desenvolvimento pessoal e institucional, com repercussões diretas na qualidade do cuidado e do ensino em Enfermagem (10).

Nesse sentido, reconhecer as características do conhecimento produzido nesse contexto pode contribuir para o reconhecimento de avanços e vazios, permitindo um olhar crítico sobre a coerência e adequação desse conhecimento para a consolidação do corpo de conhecimento da Enfermagem. Acreditamos, portanto, que se faz necessário o conhecimento da produção de conhecimento nos cursos de mestrado e doutorado, de modo sistematizado, como um importante processo de avaliação da contribuição dessa formação, destacando a convergência ciência-arte e tecnologia dirigidas a prática assistencial e de ensino em saúde e Enfermagem, implicadas em diferentes áreas tecnológicas de estudo.

Assim, o objetivo deste estudo foi conhecer algumas características do conhecimento produzido em um Curso de Mestrado em Enfermagem no Brasil, no período de 20052008 , no tocante a área temática, tipo de estudo, linhas de pesquisa e participantes do estudo.

\section{METODOLOGIA}

O presente trabalho identifica-se como uma pesquisa quantitativa de abordagem descritiva. O estudo consta de análise documental das 60 dissertações defendidas no Curso de Mestrado do Programa de Pós-graduação em Enfermagem (PEn) da Universidade Federal de Santa Catarina - UFSC, no período de janeiro de 2005 a janeiro de 2008 . O referido Programa foi criado em 1976, e tem por objetivo a capacitação de enfermeiros e profissionais da área da saúde, em nível avançado, para a produção de conhecimentos em saúde e para assumir papel de liderança no campo educacional, assistencial e político da área, defendendo o direito universal à saúde, à uma vida digna e à assistência de qualidade. O foco do Programa é o cuidado no processo de viver humano.

Os dados foram coletados mediante a leitura dos resumos das dissertações e, posteriormente, por meio da leitura do texto completo das dissertações, arquivadas no PEN/ UFSC, quando a informação não constava do resumo. Para o registro dos dados foi elaborado um instrumento para classificar as dissertações quanto à área temática do estudo (educação, cuidado à saúde, articulação ensino, cuidado e pesquisa, comunicação/ cultura/história em saúde, avaliação, tecnologias inventivas de produtos e processos de ensinar/aprender e cuidar, processo de trabalho em enfermagem e promoção a saúde), as linhas de pesquisa, classificadas de acordo com o PEN/UFSC (o cuidado e o processo de viver, ser saudável e adoecer; filosofia e ética em saúde e enfermagem; processo de trabalho em saúde; administração em enfermagem e saúde; arte, criatividade e tecnologia em saúde e enfermagem; história em enfermagem e saúde; o cotidiano e o imaginário no processo saúde-doença; educação, saúde e enfermagem), o tipo de estudo (quantitativo, qualitativo ou quanti-qualitativo) e os sujeitos participantes dos estudos. 
Os dados obtidos foram analisados através de medidas estatísticas (números absolutos e relativos) e apresentados em forma de tabelas e gráficos. Em relação às questões éticas foram observados os seguintes aspectos: autorização institucional para a coleta de dados e anonimato dos autores cujos trabalhos finais integram o presente estudo.

\section{RESULTADOS E DISCUSSÃO}

A análise das dissertações defendidas no período estudado (60) mostrou que a área temática predominantemente foi o cuidado a saúde com 26 (43,33\%) dissertações, seguida da área temática de educação com 19 (31,67\%) dissertações. As demais áreas foram abordadas em poucas dissertações e houve áreas que não foram abordadas, tais como produtos e processos de ensinar-aprender e cuidar e a área temática de avaliação (Gráfico 1). A concentração de estudos nas áreas de cuidado à saúde e de educação demonstram uma aderência dos mesmos ao trabalho cotidiano do enfermeiro, que poderíamos chamar de áreas clássicas. Isto demonstra a preocu- pação do enfermeiro por um conhecimento que aprimore sua prática, no cuidado direto ao paciente, no cuidado ao cuidador, ou na administração do ambiente, corroborando a importância da área temática no corpo de conhecimento disciplinar.

Já a temática de educação assume importante papel nesse período, concentrando grande parte dos estudos. O reconhecimento da importância dos processos educativos em suas diferentes vertentes (em saúde, formal e educação permanente) no contexto da prática de enfermagem, associado aos processos de mudança no cenário educacional brasileiro podem explicar o significativo número de estudos nessa área.

Chama a atenção à inexistência de estudos de caráter avaliativo nesse período, já que essa temática vem tendo destaque na área da saúde, especialmente quando se discute o impacto e a resolutividade das ações em saúde e, em especial, as ações de enfermagem. Sem dúvida esse é um vazio importante que requer uma revisão de investimentos em estudos, uma vez que tais estudos podem contribuir para a definição de políticas públicas, para a re-orientação das práticas em saúde e para a valorização social da profissão.

Gráfico 1. Dissertações de Mestrado em Enfermagem, segundo àreas de estudo. PEN/UFSC, 2004-2008.

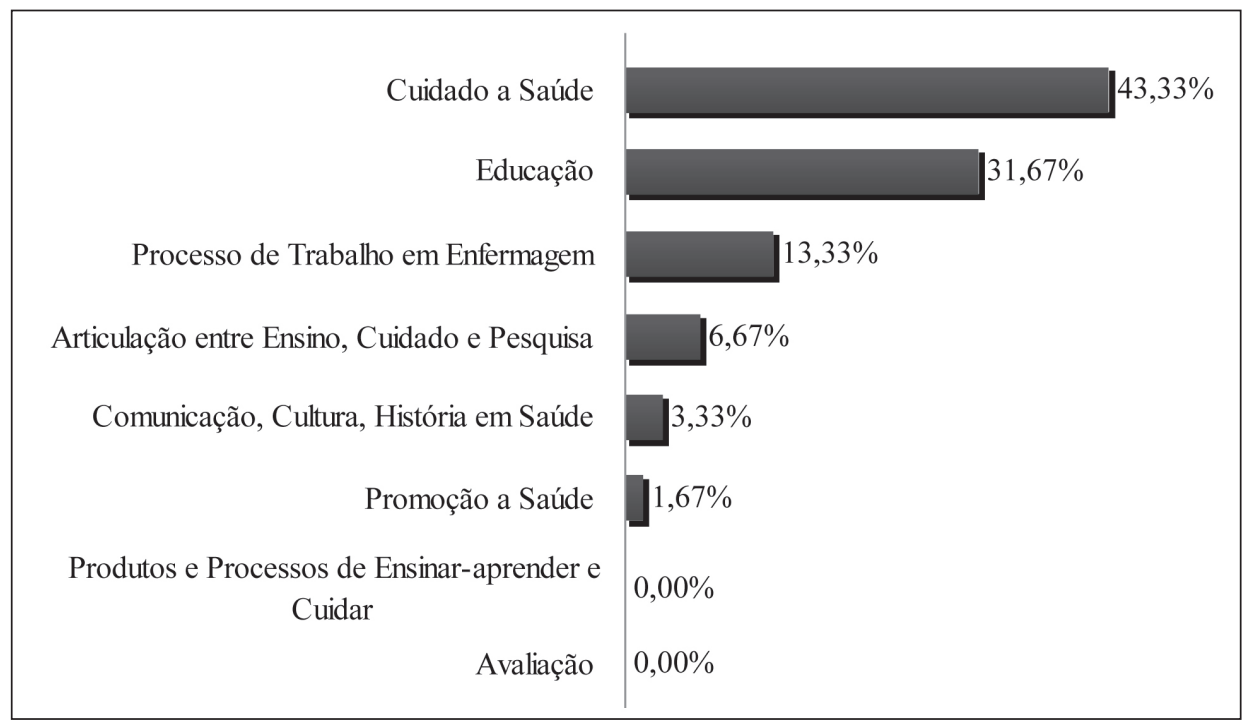


Os resultados encontrados quanto ao tipo de estudo, demonstram que as dissertações elaboradas neste período são em sua quase totalidade pesquisas do tipo qualitativa $(93,3 \%)$, seguida por estudos do tipo qualiquantitativo $(3,3 \%)$ e quantitativo $(3,3 \%)$. A predominância de estudos qualitativos corrobora uma tendência na produção do conhecimento em enfermagem no Brasil, consolidando uma perspectiva já identificada em outro estudo (11).

A natureza dos estudos qualitativos, que valorizam a subjetividade e buscam compreensão dos fenômenos tem sido uma das razões pelas quais a Enfermagem tem investido na produção do seu corpo de conhecimento a partir do paradigma naturalístico. Mais alinhado com a própria natureza das práticas de enfermagem, as abordagens qualitativas valorizam a subjetividade e a afetividade presente nas relações de cuidado. Talvez, por isso, sejam capazes de contribuir com uma compreensão mais próxima à natureza da disciplina. A predominância de estudos qualitativos também nos indica um novo processo de construção do conhecimento de enfermagem neste Curso de Mestrado, no
Brasil, evidenciando a busca por um conhecimento único para a Disciplina. Isto porque, tais estudos exigem a utilização de métodos e abordagens teóricas de outras disciplinas, na busca de uma fundamentação sólida, e a partir disto levam a diferentes dimensões na esfera da construção do conhecimento e da consolidação e proposição de novas metodologias.

Todavia, essa não se constitui em uma tendência nos estudos de Enfermagem no mundo. Estudo realizado no Chile demonstrou uma maior porcentagem de estudos quantitativos produzidos em um Curso de Mestrado (12) e o mesmo resultado foi obtido para outros países da Europa, Ásia e América do Norte (13). Em apenas alguns países, como Noruega, Suécia, Austrália, Irlanda e Reino Unido, os estudos quantitativos não foram predominantes (13).

Os resultados evidenciaram, ainda, que as Dissertações de Mestrado apresentam uma distribuição não uniforme entre as oito linhas de pesquisa (LP) do Programa, contribuindo de modo desigual para a sustentação das mesmas (Gráfico 2). Na LP "O cuidado e processo de viver, ser saudável e adoecer" fo-

Gráfico 2. Dissertações de Mestrado em Enfermagem, segundo linhas de pesquisa. PEN/UFSC, 2004-2008.

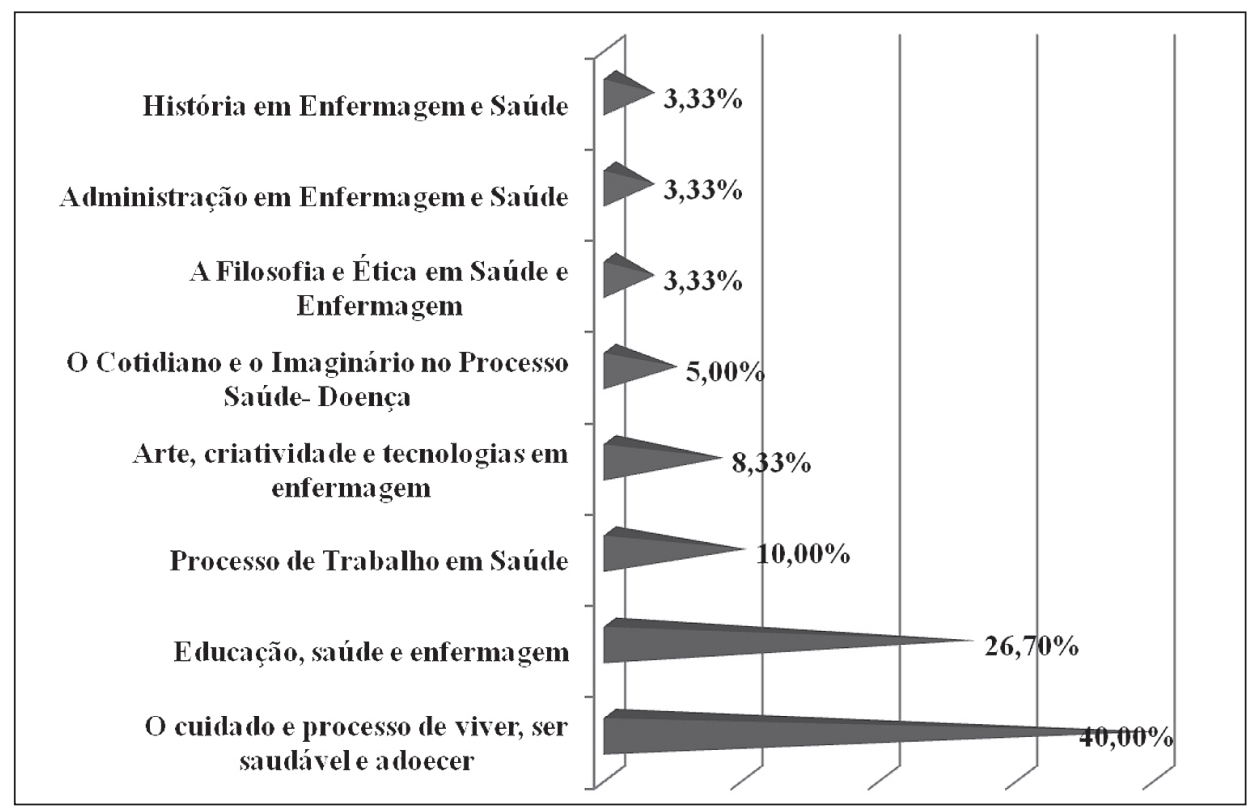


ram realizadas $24(40,0 \%)$ dissertações. Esta linha é considerada central no PEN/UFSC, já que aborda o processo de cuidado aos diferentes indivíduos, incorporando as singularidades do processo de viver humano nos diferentes etapas da vida. Significativo número de dissertações 16 (26,7\%) está vinculado a LP “Educação, saúde e enfermagem”, reunindo estudos sobre concepções políticas, filosóficas, teóricas, pedagógicas e tecnologias educacionais, sua aplicação e conseqüências na saúde e na enfermagem, bem como a dimensão educativa do trabalho em saúde e enfermagem e a educação popular e saúde. A terceira LP com maior número de dissertações foi a de "Processo de trabalho em saúde" com 6 (10,0\% das dissertações), cujos estudos tratam das inter-relações entre trabalho, saúde e cidadania nos cenários históricos sociais, bem como as dimensões estruturais, instrumentais, relacionais e subjetivos no processo de trabalho, em especial, na saúde e enfermagem. Estudos relativos ao processo de trabalho em enfermagem aparecem mais fortemente a partir do final dos anos $80 \mathrm{e}$ início dos 90 do século passado, quando as discussões político-estruturais do sistema de saúde tomam força no Brasil.
A produção científica organizada a partir de linhas de pesquisa consiste em uma importante estratégia de fortalecimento e visibilidade de uma disciplina, sendo fundamental a clara definição das mesmas nos cursos de mestrado e doutorado, já que estes constituem o lócus mais significativo de produção do conhecimento da Enfermagem (11). A partir dos resultados encontrados neste estudo podemos verificar que as três linhas de pesquisa com o maior número de dissertações produzidas podem ser consideradas como consolidadas, representando uma importante contribuição para o corpo de conhecimento da Enfermagem.

A população participante das dissertações estudadas apresenta um amplo escopo, o que demonstra a diversidade de sujeitos em diferentes situações e condições do processo de viver, para os quais se dirige ou pode se dirigir o cuidado de enfermagem. Os sujeitos que foram participantes nas dissertações estudadas foram os próprios enfermeiros e a equipe de enfermagem em 10 (16,7\%), seguido de familiares/acompanhantes/clientes (adultos, sem distinção de gênero) em 9 $(15,0 \%)$ e mulheres e gestantes em $8(13,3 \%)$ (Gráfico 3). Resultado semelhante foi encon-

Gráfico 3. Dissertações de Mestrado em Enfermagem, de acordo com os sujeitos participantes. PEN/UFSC, 2004-2008.

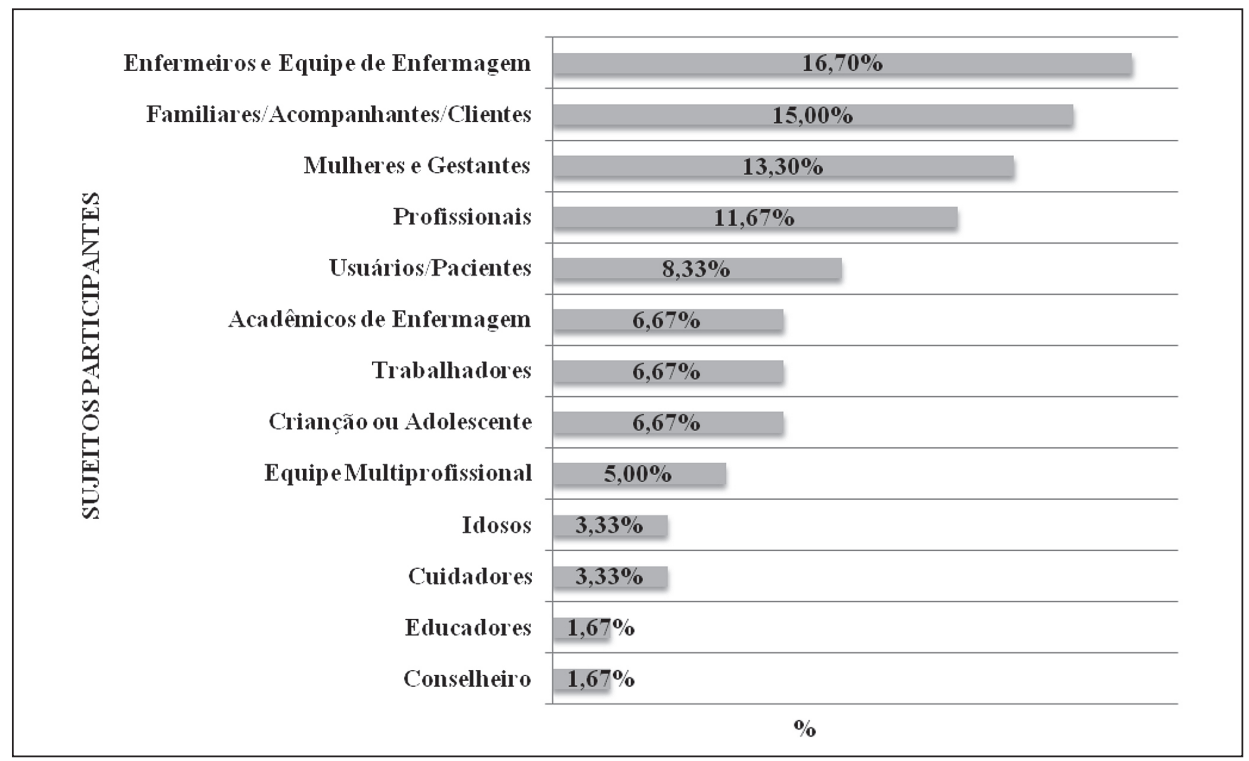


trado em outros estudos, o que demonstra uma tendência mundial $(12,13)$, inclusive o significativo número de estudos realizados com o coletivo de enfermagem. O predomínio de enfermeiros e da equipe de enfermagem como sujeitos nos estudos analisados, ao contrário de refletir uma fragilidade ou imaturidade da produção científica da Enfermagem, reflete a preocupação com a própria profissão e com a compreensão de sua prática, além de apontar para uma preocupação com a consolidação da disciplina no contexto da assistência em saúde.

A predominância de estudos realizados com familiares/acompanhantes/clientes e mulheres/gestantes aponta para uma preocupação com o cuidado de Enfermagem prestado a esse grupo, o que contribui para a compreensão das intercorrências no processo de viver humano, bem como para uma maior qualificação do cuidado prestado à clientela (14-17).

\section{CONSIDERAÇÕES FINAIS}

A análise da produção científica a partir das dissertações elaboradas em um Curso de Mestrado em Enfermagem evidencia a consolidação de um conhecimento voltado para o cuidado à saúde e a educação, por meio de estudos qualitativos, que estão distribuídos predominantemente em três linhas de pesquisa. A linha "O cuidado e processo de viver, ser saudável e adoecer" tem destaque, sendo que possui interface direta com a área temática estudada, e com a predominância dos sujeitos envolvidos nos estudos, ou seja, enfermeiros e a equipe de enfermagem, seguidos dos familiares/acompanhantes/clientes e mulheres e gestantes.

Esse trabalho também permite identificar as fragilidades e os vazios na produção do conhecimento. No tocante a temas, a ine- xistência de estudos de avaliação em saúde aparece como uma importante fragilidade, já que os mesmos podem contribuir com o estabelecimento de prioridades para a educação e a atenção em saúde e enfermagem. Com relação às linhas de pesquisa, identificou-se uma menor produção nas linhas de historia, de filosofia e ética e de administração em saúde e enfermagem. Acreditamos que estudos nessas linhas possam trazer importantes contribuições, para o conhecimento da profissão, de seus fundamentos teórico-filosóficos e para a melhoria da gestão do cuidado de enfermagem (15-17). Temas esses relevantes para a construção e fortalecimento da disciplina. Relativamente aos participantes, o menor número de estudos envolvendo cuidadores e idosos também se apresenta como uma fragilidade, tendo em vista o perfil epidemiológico da população brasileira, que aponta para o aumento da expectativa média de vida.

O estudo nos revela um panorama da produção científica em um curso de mestrado de enfermagem, destacando suas fortalezas, como seu alinhamento as novas abordagens em saúde e o cuidado em saúde, os quais podem contribuir para pensar novos modelos assistenciais e organizacionais da enfermagem e da saúde. Também evidencia as fragilidades, como o desequilíbrio de sua produção em relação às linhas de pesquisa $\mathrm{e}$ os grupos para os quais se dirigem. Assim, o presente estudo aponta para a necessidade de re-alinhamento da produção científica nesses diferentes aspectos, de modo a contribuir para a consolidação do conhecimento científico de enfermagem no atendimento aos requerimentos de cuidado em saúde da população. Tambem, evidencia a importância de outros estudos que identifiquem as fortalezas e debilidades da produção científica em enfermagem, de modo a contribuir com o estabelecimento de prioridades em pesquisa no contexto disciplinar. 


\section{REFERÊNCIAS}

1. Meehan TC. Careful Nursing: a model for contemporary nursing practice. J Adv Nurs. 2003; 44 (1): 99-107.

2. Riehl JP, Roy C. History of Nursing Models. In: Riehl JP, Roy C. Conceptual Models Nursing Practice. New York: Appleton-Century-Crofts, 1974.

3. Villalobos MMD. Enfermería: desarrollo teórico e investigativo. Colombia: Universidad Nacional de Colombia, 1998.

4. Carraro TE. Sobre Teorias e Marco Conceitual: sua influência na metodologia de assistência. En: Carraro TE, Westphalen MAE. Metodologias para assistência de enfermagem: teorização, modelos e subsídios para a prática. Goiânia: AB; 2001. p. 29-37.

5. Carraro TE (org.). Investigación Cualitativa en Enfermería: contexto y bases conceptuales. Washington D.C.: Série Paltex Salud y Sociedad, Organización Panamericana de la Salud; 2008.

6. Fawcett J. Knowledge Contemporary Nursing Knowledge: analysis and evolution of nursing models and theories. 2 ed. Philadelphia: F. A. Davis Company; 2005.

7. Adue IM, Chervo MA, Carraro TE, Prado MLd. Las Controversias entre Cuantificación y Cualificación en Investigación. En: Prado MLdo, Souza MdeL, Carraro TE (org.). Investigación Cualitativa en Enfermería: contexto y bases conceptuales. Série Paltex Salud y Sociedad 2000 $\mathrm{N}^{\circ}$ 9, Washington D.C.: Organización Panamericana de la Salud; 2008.

8. Santos TCF, Gomes MdaLB. Nexos entre pós-graduação e pesquisa em Enfermagem no Brasil. Rev Bras Enferm. 2007; 60 (16): 91-5.
9. Baptista SS, Barreira IA. Enfermagem de nível superior no Brasil e vida associativa. Rev Bras Enferm. 2006; 59 (n.spe): 411-416.

10. Prado MLdo, Gelbcke FL. Produção de Conhecimento em Enfermagem no Brasil: as temáticas de investigação. Rev Bras Enferm. 2001; 54 (1): 34-42.

11. Erdmann AL, Silva IA, Rodrigues RAP, Fernandes JD, Vianna LAC, Lopes MJM, Santos RS, Araújo TL de. Teses produzidas nos programas de Pós-Graduação em Enfermagem de 1983 a 2001. Rev Esc Enferm USP. 2005; 39 (n.spe.): 497-505.

12. Paravic KT, Burgos MM. Evaluación de calidad de resúmenes de tesis de un programa de magíster en enfermería. Cienc. enferm. 2009; 15 (3): 55-68.

13. Polit DF, Beck T. International Differences in Nursing Research. J Nurs Scholarsh. 2009; 41 (1): 44-53.

14. Leopardi M, Nietzsche E. O Saber da Enfermagem como Tecnologia: A Produção de Enfermeiros Brasileiros. Florianópolis-SC, UFSC. Texto contexto-enferm. 2000; (9):129-150.

15. Baptista SS, Barreira, IA. Enfermagem de nível superior no Brasil e vida associativa. Rev Bras Enferm. 2006; 59 (n.spe): 411-416.

16. Padilha MICS, Borenstein MS, Maia AR, Guedes JAD, Lessmann JC, Machado CA. Uma história de sucesso: 30 anos da Pós-Graduação em Enfermagem da UFSC. Texto contexto-enferm. 2006; 15 (n. spe): 20-30.

17. Rodrigues RAP, Erdmann AL, Silva IA, Fernandes JD, Araújo TL, Vianna. LAC, Santos RS, Lopes MJM. Educação do doutorado em enfermagem no Brasil. Rev Lat Am Enfermagem. 2008; 16 (4):665-671. 